\title{
Cytotoxicity of Three New Triazolo-pyrimidine Derivatives against the Plant Trypanosomatid: Phytomonas sp. Isolated from Euphorbia characias
}

\author{
Rosa Magán, Clotilde Marín' Juan M Salas*, Mario Barrera-Pérez**, Maria J Rosales, \\ Manuel Sánchez-Moreno/ ${ }^{+}$
}

\begin{abstract}
Departamento de Parasitología, Instituto de Biotecnología *Departamento de Química Inorgánica, Facultad de Ciencias, Universidad de Granada, Severo Ochoa s/n, E-18071 Granada, España **Laboratorio de Parasitología, Centro de Investigaciones Regionales, Universidad Autónoma de Yucatán, Mérida, México

There is no effective chemotherapy against diseases caused by Phytomonas sp., a plant trypanosomatid responsible for economic losses in major crops. We tested three triazolo-pyrimidine complexes [two with Pt(II), and another with Ru(III)] against promastigotes of Phytomonas sp. isolated from Euphorbia characias. The incorporation of radiolabelled precursors, ultrastructural alterations and changes in the pattern of metabolite excretion were examined. Different degrees of toxicity were found for each complex: the platinun compound showed an inhibition effect on nucleic acid synthesis, provoking alterations on the levels of mitochondria, nucleus and glycosomes. These results, together with others reported previously in our laboratory about the activity of pyrimidine derivatives, reflect the potential of these compounds as agents in the treatment of Phytomonas $s p$.
\end{abstract}

Key words: Phytomonas sp. - triazolo-pyrimidine complexes - chemotherapeutic agents

The name Phytomonas was proposed by Donovan (1909) to designate a trypanosome discovered by Lafont in the same year and initially named Leptomonas davidi. Phytomonas affects more than 100 plant species in about 12 families, causing diseases in plants of substantial economic interest, such as "sudden wilt" in the oil palm and "Hartrot" disease in the coconut palm, among others (Dollet 1984). Phytomonas sp. are distributed primarily in tropical and subtropical zones, although they have been described in all continents except the Antarctic (Camargo \& Wallace 1994). Although there is a certain knowledge about the biology of this protozoan (Camargo 1999), apart from studies aconducted in our laboratory (Luque et al. 2000a, b), little information is available on its control. To finding of an effective chemotherapy against Phytomonas spp. has not only a direct economic interest, but since these parasites present metabolic routes similar to those of other Trypanosomatidae pathogenic to humans and animals, they represent also a potential biological model (Uttaro et al. 1999, Magán et al. 2004).

We have previously demonstrated the biological activity of some metal complexes of triazolo-pyrimidine derivatives against Phytomonas staheli, Leishmania donovani, and Trypanosoma cruzi, inhibiting the synthesis of DNA, RNA, and proteins and inducing severe ultrastructural damage (Luque et al. 2000a, b,).

\footnotetext{
${ }^{+}$Corresponding author. Fax: +34-9-58-243174. E-mail: msanchem@ugr.es

Financial support: Universidad de Granada (Spain), grant: BIO2000-1429

Received 25 November 2003

Accepted 3 August 2004
}

The metal complexes with triazolo-pyrimidine derivatives are ligands that could mimic purines. These compounds have already been reported as growth inhibitors of some microorganisms (reviewed in Fischer 1993), and tumoral lineages (Chae et al. 1995, Quintero et al. 1999), by inhibition of DNA synthesis. Their potential in the treatment of neurodegenerative disorders such as Parkinson's disease has also been tested (Baraldi 1996).

In this work, we seek to advance in the development of an effective chemotherapy by testing three new triazolopyrimidine derivatives on promastigotes of Phytomonas isolated from Euphorbia characias, investigating their possible mechanism of action.

\section{MATERIALS AND METHODS}

Parasites - The promastigote forms of $P$. characias were isolated from E. characias in Montpellier (France) and kindly provided by Dr M Dollet. The flagellates were cultured in Grace's medium at $28^{\circ} \mathrm{C}$ in plastic Roux flasks (Corning, Washington, DC, US) using an initial concentration of $5 \times 10^{4}$ cells $/ \mathrm{ml}$. On the fourth day of culture, the parasites were centrifuged at $1500 \mathrm{~g}$ for $10 \mathrm{~min}$ and the pellet was washed twice in PBS. Proliferation of the parasites was monitored by counting in a Neubauer chamber.

Compounds assayed - The compounds used were termed; SPIV: Cis $\left[\mathrm{PtCl}_{2}(7 \mathrm{HtpO})_{2}\right] \cdot 2 \mathrm{H}_{2} \mathrm{O}$; SPV: $\mathrm{PtCl}_{2}$ $(5 \mathrm{HtpO})_{2} \cdot 2 \mathrm{H}_{2} \mathrm{O}$ and SPVI: $\mathrm{RuCl}_{3}(7 \mathrm{HtpO})_{2} \cdot 2 \mathrm{H}_{2} \mathrm{O}$, and were synthesized by Salas et al. (2001).

The ligand $7 \mathrm{HtpO}$ corresponds to 4,7 dihydro -7-oxo$[1,2,4]$ triazolo $[1,5-\mathrm{a}]$ pyrimidine and $5 \mathrm{HtpO}$ to 4,5 dihydro -5- oxo-[1,2,4] triazolo [1,5-a] pyrimidine (Fig. 1).

Dimethyl sulfoxide was used as solvent of the compounds and the final concentration in the experiments was $0.01 \%(\mathrm{v} / \mathrm{v})$, which was previously shown not to interfere with parasite growth (Mesa-Valle et al. 1996). 
<smiles>O=c1ccn2ncnc2[nH]1</smiles>

Fig. 1: structure of the newly synthesized $[1,2,4]$ triazolo $[1,5-\mathrm{a}]$ pyrimidine derivatives

Macromolecule synthesis by P. characias - The effect of the compounds on the parasite's biosynthesis of nucleic acids and proteins was evaluated by the incorporation of 6- $\left[{ }^{3} \mathrm{H}\right]$ thymidine $(26-30 \mathrm{Ci} / \mathrm{mmol}), 5-\left[{ }^{3} \mathrm{H}\right]$ uridine $(25-30 \mathrm{Ci} /$ $\mathrm{mmol}$ ) or $4,5-\left[{ }^{3} \mathrm{H}\right]$ leucine $(35-70 \mathrm{Ci} / \mathrm{mmol})$. All these radiolabelled compounds were obtained from the Radiochemical Centre (London, UK). Cultures containing $5 \times 10^{6}$ cells/ $\mathrm{ml}$ (total volume $200 \mathrm{ml}$ ) were centrifuged, the pellet was resuspended in $1 \mathrm{ml}$ of culture medium and compounds was added to give the final concentration of $5 \mu \mathrm{M}$. After incubation at $28^{\circ} \mathrm{C}$ for $30 \mathrm{~min}$, the medium was replaced by a fresh one containing $5 \mu \mathrm{g} / \mathrm{ml}$ of each radioactive analog. At $0,45,75$ and $135 \mathrm{~min}$, the cultures were centrifuged, and the pellet, after three washes with cold medium, was precipitated with $10 \%$ trichloroacetic acid for 2 $\mathrm{h}$ at $4^{\circ} \mathrm{C}$. The precipitates were filtered through Whatman $\mathrm{GF} / \mathrm{c}$ filters, washed with $5 \%$ trichloroacetic acid and then with $10 \%$ ethanol, and dried at $60^{\circ} \mathrm{C}$ under infrared lamps. Once dried, the filters were submerged in scintillation cocktail \{2,5-diphenyloxazole [PPO], $4 \mathrm{~g}$; 1,4-bis(5-phenyloxazoly)benzene [POPOP], $0.1 \mathrm{~g}$; toluene, $1000 \mathrm{ml}$ \}, and the radioactivity incorporated was determined with a betaspectrometer (Beckman model LS 6000TA, Palo Alto, CA, US). Triplicates were made of each assay and the mean \pm SD was determined (Mesa-Valle et al. 1996).

Ultrastructural alterations - The parasites $\left(5 \times 10^{5}\right.$ cells $/ \mathrm{ml}$, volume total $200 \mathrm{ml}$ ), were cultured in Grace's medium, containing $5 \mu \mathrm{M}$ of the compounds. After $72 \mathrm{~h}$, the parasites were centrifuged at $1500 \mathrm{~g}$ for $10 \mathrm{~min}$, washed in fresh medium, and fixed for $2 \mathrm{~h}$ in a $2.5 \%(\mathrm{v} / \mathrm{v})$ solution of cacodylate-buffered $0.1 \mathrm{M}$ glutaraldehyde containing $0.1 \mathrm{M} \mathrm{CaCl}_{2}$ and $0.9 \% \mathrm{NaCl}$. The fixed cells were washed in the same buffer and postfixed in isotonic saline solution containing $1 \%(\mathrm{v} / \mathrm{v})$ osmiun tetroxide and $0.08 \%$ potassium fluorocyanide. After postfixation the cells were washed in buffer, dehydrated by acetone washes, and embedded in Epon (Luque et al. 2000b). Thin sections were stained with uranyl acetate and lead citrate and exmined with an EMCIO Zeiss transmission electron microscope.

Metabolite excretion - After the same treatment as described above, the supernatants were collected to determine the excreted metabolites by nuclear magnetic resonance spectroscopy $\left({ }^{1} \mathrm{H}-\mathrm{NMR}\right)$ (Sánchez-Moreno et al. 1992). The chemical displacements, expressed in parts per million ( $\mathrm{ppm}$ ) using sodium 2,2dimethyl-2-silapentane-5sulfonate as the reference signal, allowed the identifica- tion of the metabolites, which were consistent with those described by Sánchez-Moreno et al. (1992). In all cases, triplicates were made of each experiment.

\section{RESULTS}

Fig. 2 shows the action of the three triazolo-pyrimidine derivatives on the incorporation of the radiolabelled precursors by the $P$. characias promastigotes. The incorporation of $6-\left[\mathrm{H}^{3}\right]$ thymidine was inhibited after $135 \mathrm{~h}$ in $79.3 \%$ and $83.1 \%$ for, respectively, SPIV and SPV and in $26.5 \%$ for SPVI (Fig. 2A). SPIV and SPV did not interfere with the incorporation of $4,5-\left[\mathrm{H}^{3}\right]$-leucine, while SPVI led to an inhibition of $26 \%$ after the same was the only compounds that inhibited (Fig. 2B). The incorporation of 5$\left[\mathrm{H}^{3}\right]$-uridine by the parasite was inhibited after $135 \mathrm{~h}$ in the range of $25 \%$ to $37.4 \%$ for the three compounds (Fig. 2C).
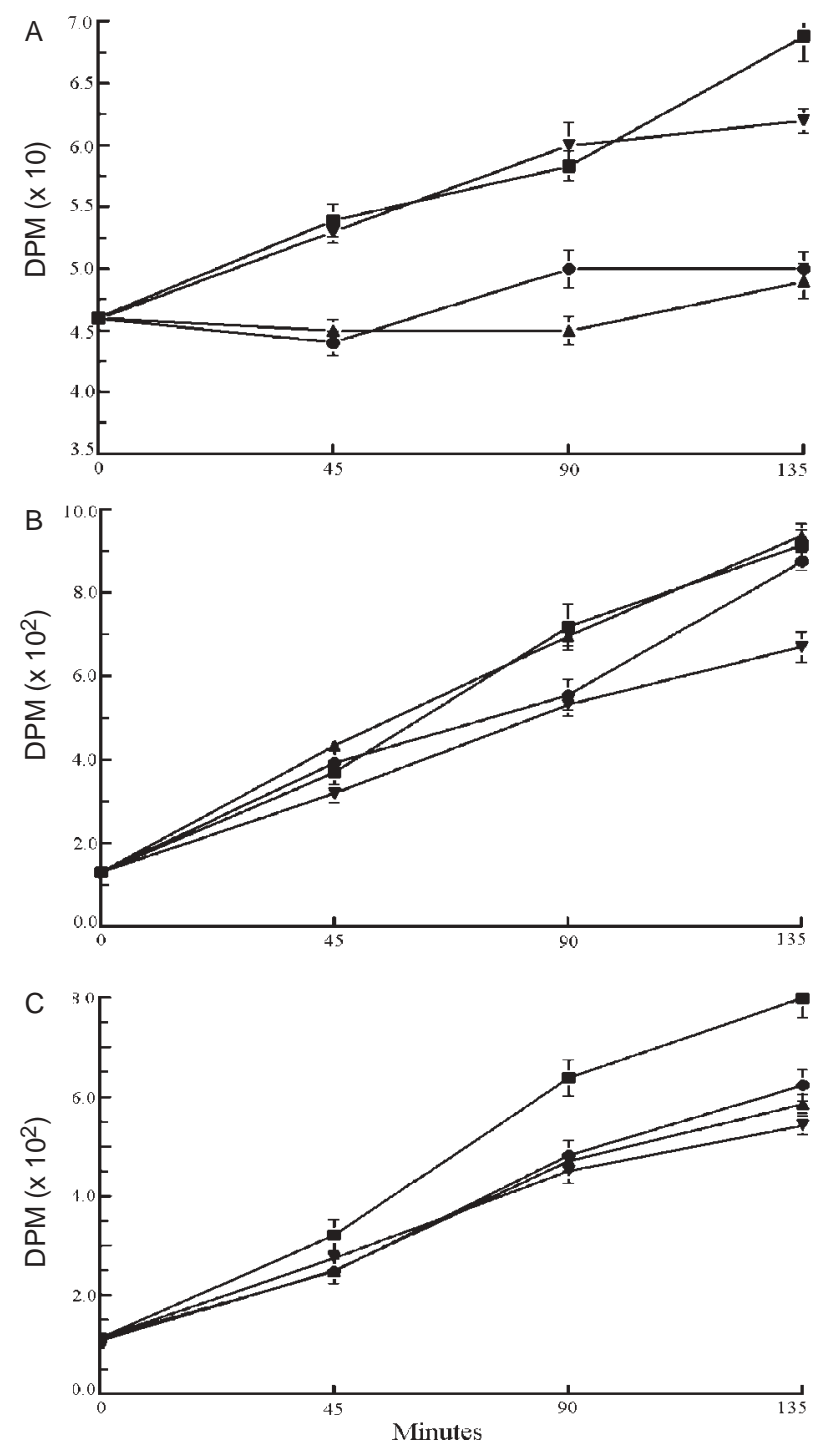

Fig. 2: effect of the triazolo-pyrimidine derivatives on the incorporation of radiolabelled precursors $6-\left[{ }^{3} \mathrm{H}\right]$ thymidine $(\mathrm{A}), 4.5$ $\left[{ }^{3} \mathrm{H}\right]$ leucine $(\mathrm{B})$ and $5-\left[{ }^{3} \mathrm{H}\right]$ uridine $(\mathrm{C})$ by promastigotes of Phytomonas characias. All compounds were tested at $5 \mu \mathrm{M}$; con-

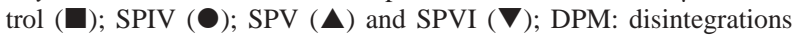
per min 
The ultrastructure of $P$. characias promastigotes is shown in Fig. 3A, revealing their principal organelles and cell organization typical of members of the family Trypanosomatidae (Fernadez-Becerra et al. 1997).

Fig. 3B shows the effect of $5 \mu \mathrm{M}$ SPIV after three days of treatment. The cell is swollen and roundish. The cytoplasm has little electrodensity and has a granular aspect with many vacuoles, most of which are apparently empty. Glycosomes are altered, less electrodense and very swollen. The mitochondrion is swollen, with no mitochondrial cryptal visible. The kinetoplast has lost its normal com- pact structure. The nucleus lacks a uniform nucleolus but chromatin accumulations were observed associated with the nuclear membrane, with discontinuous distribution around the nucleus.

Fig. 3C reveals the alterations produced by SPV in promastigote forms of $P$. characias. The cytoplasm appears less dense, and large empty vacuoles and cytoplasmatic disorganization are evident. Alterations in the glycosomes are evident, being granulose and swollen. The mitochondrion is also swollen and the kinetoplast has a disorganized structure.
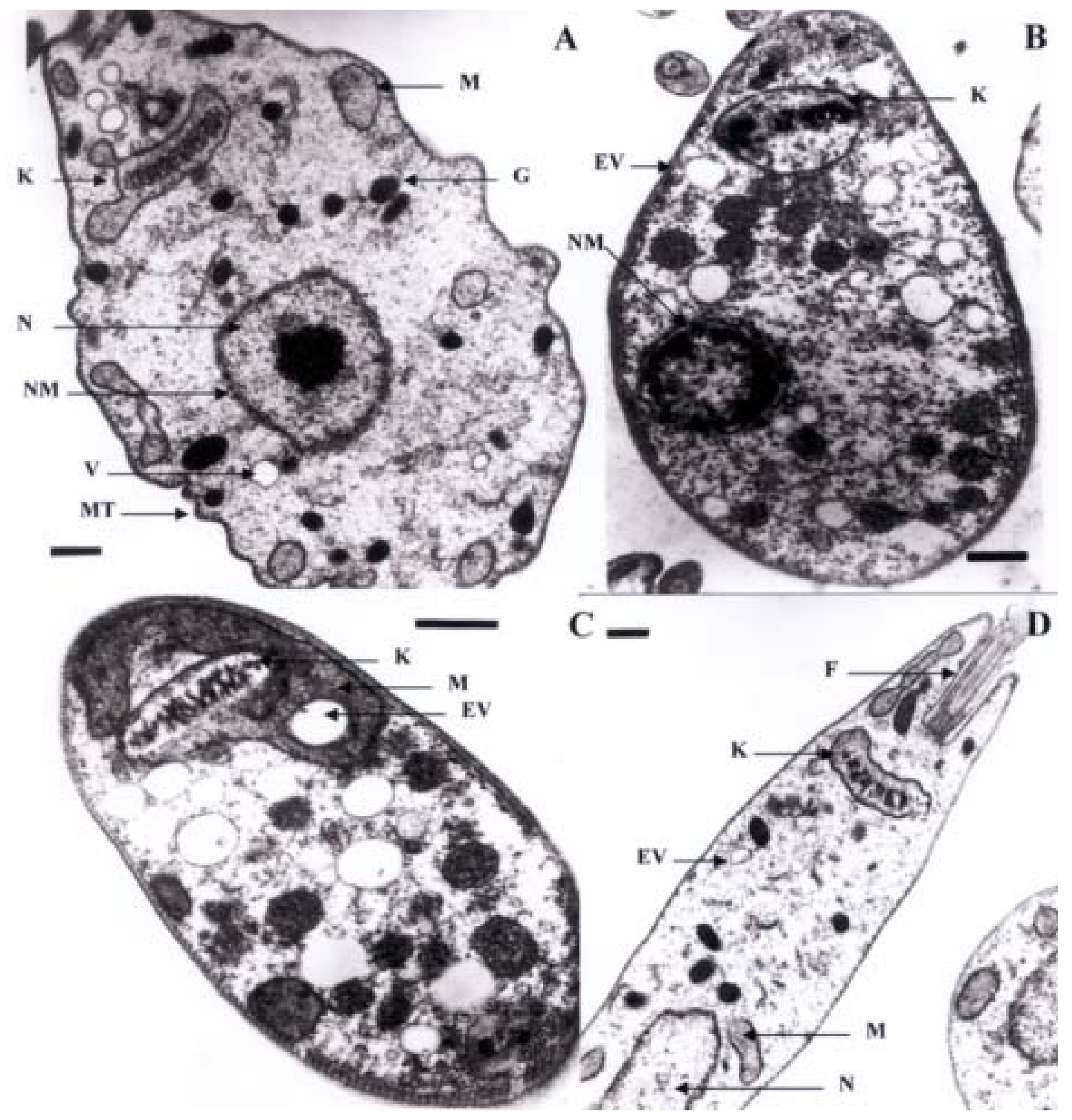

Fig. 3: ultrastructural effect of triazolo-pyrimidine derivatives $(5 \mu \mathrm{M})$ on promastigotes of Phytomonas characias cultured in Grace's medium for 72 h. A: control; B: SPIV; C: SPV; D: SPVI; EV: empty vacuoles; G: glycosome; K: kinetoplast; M: mitochondrion; MT: microtubules; N: nucleus; NM: nuclear membrane; V: vacuoles. Bars $=0.583 \mu \mathrm{m}$ 
Fig. 3D shows the alterations in $P$. characias treated with SPVI. The parasite does not appear swollen on being treated with SPVI, but the cytoplasm appears to be less electrodense than in the control parasites. The nucleus seems also to be altered, with reduction of its contents. Also, alteration in the kinetoplast can be observed, with a less compact organization of the kDNA.

The main metabolites excreted by $P$. characias are, in ascending order: acetate, ethanol, succinate, glycine and, in less quantities, L-alanine and lactate (Fig. 4A).

Figs 4B, C, D show the spectra that resulted from the treatment of $P$. characias with of the triazolo-pyrimidine compounds. The SPIV and SPV at $5 \mu \mathrm{M}$ (Figs 4B, C) strongly inhibited all the metabolites excreted. The spectrograph corresponding to the effect of SPV (Fig. 4C) indicates an additional peak (at $2.3 \mathrm{ppm}$ ) not detected in the spectrum of untreated parasites, which was identified as pyruvate. SPVI notably inhibited only the excretion of acetate (roughly 25\%) (Fig. 4D).

\section{DISCUSSION}

The chemotherapeutic capacity of the triazolo-pyrimidines appear promising for due to their similarities to purines, prompting us to test the three compounds against microorganisms. Furthermore, these compounds have been used as molecular models to study the interactions of the metal ions with nucleic acids (Ram et al. 1992). The two derivatives that carry the $\mathrm{Pt}(\mathrm{II})$ in their structures (SPIV and SPV) inhibit the incorporation of 6- $\left[{ }^{3} \mathrm{H}\right]$ - thymidine by $P$. characias, indicating interference in the DNA synthesis. This effect may be due to the fact that, resembling pyrimidines, these derivatives could intercalate in DNA, altering its secondary structure. This fact is confirmed by the electron microscopy results, which show
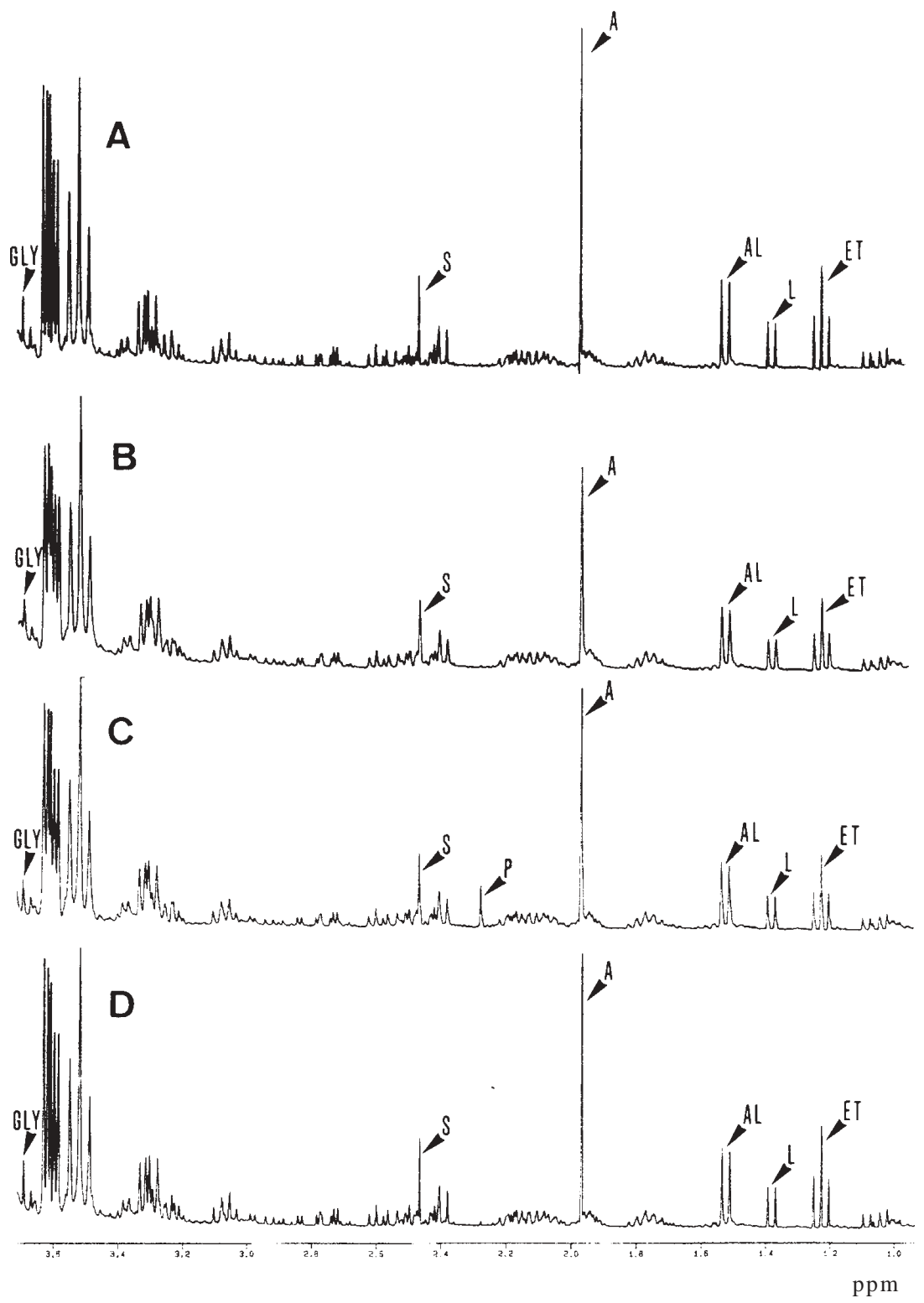

Fig. 4: $\left[{ }^{1} \mathrm{H}\right]$ NMR study of the production of ethanol (ET), lactate (L), L-alanine (AL), acetate (A), pyruvate (P), succinate (S), glycine (GLY) by promastigote of Phytomonas characias treated with $5 \mu \mathrm{M}$ of the triazolo-pyrimidine derivatives; A: control; B: SPIV; C: SPV; D: SPVI 
that the organization of both DNA and the chromatin are altered.

SPVI also inhibits, though to a lesser degree, DNA synthesis and, like the other two derivatives, affects genetic material. A decrease in the incorporation of 5- $\left[{ }^{3} \mathrm{H}\right]-$ uridine, indicates a disturbance of RNA synthesis induced by SPVI. These data, together with those previously gathered by our group (Luque et al. 2000b, Magán et al. 2004), point to the possibility that one of the possible targets of the triazolo-pyrimidine derivatives is nucleic acid synthesis. Leucine incorporation was also visibly diminished by treatment with SPVI, suggesting that this compound can act directly on protein synthesis (Zollner 1993), or can indirectly affect ribosomal factors.

Although the ultrastructure analysis does not enable us to define the mode of action of these compounds against $P$. characias, the alterations observed reflect the beginning of the protozoa's death. In a same field (data not shown), parasites showed very different degrees of alteration. In this experiment, we use a concentration of 5 $\mu \mathrm{M}$, because this value is roughly 10 -fold lower than that of $\mathrm{IC}_{50}$ found in prior studies (Salas et al. 2001).

In conclusion the three compounds seem to induce several alterations in the mitochondrion, nucleus and glycosomes of these kinetoplastids. SPIV and SPV provoked very clear alterations not only in the cytoplasm and organelles, but also in the cell nucleus (mainly SPV). Both compounds provoked similar alterations which were more patent than those caused by SPVI. Swelling of the parasites was observed only after treatment with SPIV and SPV. These complexes could change the osmotic pressure of the parasite, forcing it to regulate the pressure with respect to the culture medium. Treatment with SPVI appears to affect primarily the nucleus although to a lesser degree it disrupts the normal structure of the kinetoplast. Due to this results, further studies will be necessary to determine if this alterations were induced by these compounds.

These effects unquestionably alter the energy metabolism of the promastigote forms of $P$. characias, as confirmed by the biochemical data gathered in the present work. Change in the excretion of different metabolites is of great use in elucidating the toxic activity of these compounds, since the catabolism of Phytomonas in general follows a pattern common among the species or isolates studied (Sánchez-Moreno et al. 1992, Fernández-Becerra et al. 1997). In the present work, the main metabolites excreted by $P$. characias were acetate, ethanol, succinate, and glycine, in accordance with previous results. Treatment with SPIV or SPV inhibited the excretion of these catabolites. The reduction of glycine and succinate may signal an effect on the glyoxalate cycle operating in Phytomonas sp. (Sánchez-Moreno et al. 1992), which may explain pyruvate as a new excreted metabolite, as occurs with SPV. The decline in acetate may also be due to direct impact on acetate synthetase, particularly SPVI, which interferes with protein synthesis. This hypothesis is reinforced by electron microscopy data, which indicates a loss in the functionality of the mitochondrion, the organelle where succinate and acetate are formed by these parasites (Sánchez-Moreno et al. 1992).
From the data accumulated in the present work and prior studies (Luque et al. 2000a, b), we conclude that the triazolo-pyrimidine derivatives represent a potential chemotherapy against diseases caused by trypanosomes. As an added advantage, treatment of J-7742 macrophages with $100 \mu \mathrm{M}$ of the derivatives for $72 \mathrm{~h}$ led to no toxicity to the mammalian cells (Luque et al. 2000a, b). Nevertheless, much work remains to be done in the study of these complexes to gain a full understanding of their functioning, for example, focusing in more detail on the ultrastructural changes induced by these derivatives.

\section{REFERENCES}

Baraldi PG, Cacciari B, Spalluto G, Villatoro MJ, Zocchi C, Dionisotti S, Ongini E 1996. Pyrazolo (4,3-E)-1, 2, 4triazolo $(1,5-\mathrm{C})$ pyrimidine derivatives-potent and selective A(2A) Adenosine antagonists. J Med Chem 39: 11641171.

Camargo EP 1999. Phytomonas and other trypanosomatid parasites of plants and fruit. Adv Parasitol 42: 29-112.

Camargo EP, Wallace FG 1994. Vectors of plant parasites of the genus Phytomonas (Protozoa, Zoomatigophorea, Kinetoplastidia). In Advances in Disease Vector Research, Spring-Verlang, New York, p. 333-359.

Chae MY, Swenn K, Kanugula S, Dolan ME, Pegg AE, Moschel RC 1995. 8-Substituted O-6-benzylguanine, substituted 6(4)-(benzyloxy) pyrimidine, and related derivatives as inactivators of human O-6-alkylguanine-DNA Alkyltransferase. J Med Chem 38: 359 -365.

Dollet M 1984. Plant diseases caused by flagellate protozoa (Phytomonas). Annu Rev Phytopathol 22: 115-132.

Donovan C 1909. Kala-azar in Madras, especially with regard to its connexion with the dog and the bug (Conorrhinus). Lancet 177: 1495-1496.

Fernández-Becerra C, Sánchez-Moreno M, Osuna A, Opperdoes FR 1997. Comparative aspects of energy metabolism in plant trypanosomatids. J Eukaryot Microbiol 44: 523-529.

Fischer G 1993. 1,2,4-triazolo (1,5-alpha) pyrimidines. Adv Heterocycl Chem 57: 81-86.

Luque F, Fernández-Ramos C, Entrala E, Rosales MJ, Navarro JA, Romero MA, Salas-Peregrín JM, Sánchez-Moreno M 2000a. In vitro evaluation of newly synthesised $[1,2,4]$ triazolo [1,5a] pyrimidine derivatives against Trypanosoma cruzi, Leishmania donovani, and Phytomonas staheli. Comp Biochem Physiol Part C 126: 39-44.

Luque F, Fernández-Ramos C, Entrala E, Rosales MJ, Marín C, Salas JM, Navarro JA, Sánchez-Moreno M 2000b. Biochemical and ultrastructural alterations caused by newly synthesized 1, 2, 4-triazolo [1,5a] pyrimidine derivatives against Phytomonas staheli (Trypanosomatidae). Toxicol In Vitro 14: 487-495.

Magán R, Marín C, Rosales MJ, Barrera MA, Salas JM, Sánchez-Moreno M 2004. Activities of Pt(II) and Ru(III) Triazole-Pyrimidine complexes against Trypanosoma cruzi and T. brucei brucei. Pharmacology 70: 83-90.

Mesa-Valle CM, Castilla-Calvente J, Sánchez-Moreno M, Moraleda-Lindez V, Barbe J, Osuna A 1996. Activity and mode of action of acridine compounds against Leishmania donovani. Antimicrob Agents Chemother 40: 684-690.

Quintero A, Pelcastre A, Solano JD, Guzmán A, Diaz E 1999. Antitumoral activity of new pyrimidine derivatives of sesquiterpenelactones. J Pharm Sci 2: 108-112.

Ram VJ, Haque N, Guru PY 1992. Chemotherapeutic agents. XXI Synthesis of n-deficient pyrimidines as leishmanicides. 
Eur J Med Chem 27: 851-856.

Salas JM, Quirós M, Abul Haj M, Magán R, Marin C, SanchezMorenoM, Faure R 2001. Activity of Pt(II) and Ru(III) triazolopyrimidine complexes against parasites of the genus Leishmania, Trypanosomes and Phytomonas. MetalBased Drugs 8: 119-124.

Sánchez-Moreno M, Lasztity D, Coppens I, Opperdoes FR 1992. Characterizacion of carbohydrate metabolism and demostration of glycosomes in a Phytomonas sp. isolated from Euphorbia characias. Mol Biochem Parasitol 54: 185200.

Uttaro AD, Mirkin N, Rider MH, Michels M, Opperdoes FR 1999. Phytomonas sp. A model of trypanosomatid metabolism and drug. Mem Inst Oswaldo Cruz 94: C19.

Zollner P 1993. Handbook of Enzyme Inhibitors, VCH, New York, p. 158-161. 